University of Wollongong

Research Online

Faculty of Engineering and Information

Faculty of Engineering and Information

Sciences - Papers: Part A

Sciences

$1-1-2006$

\title{
Validation of the Geant4 bremsstrahlung models: First results
}

S Chauvie

INFN Sezione di Genova, Italy

S Guatelli

INFN Sezione di Genova, susanna@uow.edu.au

B Mascialino

INFN Sezione di Genova, barbara.mascialino@ki.se

L Pandola

Infn Laboratorio Nazionale Del Gran Sasso

M G. Pia

INFN Sezione di Genova

See next page for additional authors

Follow this and additional works at: https://ro.uow.edu.au/eispapers

Part of the Engineering Commons, and the Science and Technology Studies Commons

Research Online is the open access institutional repository for the University of Wollongong. For further information contact the UOW Library: research-pubs@uow.edu.au 


\title{
Validation of the Geant4 bremsstrahlung models: First results
}

\begin{abstract}
A project is in progress for a systematic, rigorous validation of Geant 4 electromagnetic physics. This paper presents the first results concerning the validation of Geant4 bremsstrahlung models against experimental data.
\end{abstract}

Keywords

results, first, validation, models, geant4, bremsstrahlung

Disciplines

Engineering | Science and Technology Studies

Publication Details

Chauvie, S., Guatelli, S., Mascialino, B., Pandola, L., Pia, M. G., Rodrigues, P. \& Trindade, A. (2006).

Validation of the Geant4 bremsstrahlung models: First results. In B. Phlips (Eds.), IEEE Nuclear Science Syposium Conference Record (pp. 1511-1515). New Jersey, United States: IEEE.

\section{Authors}

S Chauvie, S Guatelli, B Mascialino, L Pandola, M G. Pia, P Rodrigues, and A Trindade 


\title{
Validation of Geant4 Bremsstrahlung models: first results
}

\author{
S. Chauvie, S. Guatelli, B. Mascialino, L. Pandola, M. G. Pia, P. Rodrigues, A. Trindade
}

\begin{abstract}
A project is in progress for a systematic, rigorous validation of Geant4 electromagnetic physics. This paper presents the first results concerning the validation of Geant4 Bremsstrahlung models against experimental data.
\end{abstract}

Index Terms- Geant4, Monte Carlo, simulation, Bremsstrahlung, validation.

\section{INTRODUCTION}

$\mathrm{G}$ EANT4 [1], [2] is an object oriented toolkit for the simulation of particle interactions with matter. It provides advanced functionality for all the domains typical of detector simulation: geometry and material modelling, description of particle properties, physics processes, tracking, event and run management, user interface and visualisation.

A peculiar feature of Geant4 is its wide physics coverage, with the provision of an ample variety of models. A systematic, rigorous validation of Geant 4 physics models is essential to evaluate their accuracy, which in turn determines the reliability of user applications; a large-scale, long-term project is in progress to document the validation of Geant4 electromagnetic models against experimental data: a first study [3] addressed the cross section of photon processes and charged particles ranges and stopping powers; this paper concerns a study of Geant4 Bremsstrahlung models against reference experimental data.

Different models for the simulation of the electron Bremsstrahlung are available in Geant4 Standard and Low Energy Electromagnetic packages.

The Low Energy Electromagnetic package [4] includes two processes for the simulation of electron Bremsstrahlung: G4LowEnergyBremsstrahlung [5], which is based on the EEDL [6] Livermore Library for the calculation of cross sections and provides three alternative angular distribution models ( $2 B N, 2 B S$ and Tsai) [7], the last one equivalent to the algorithm in GEANT 3 [8] and in Geant4 Standard

Manuscript received November 24, 2006.

S. Chauvie is with the Azienda Ospedaliera Santa Croce e Carle, Cuneo and with INFN Sezione di Torino, Italy, (e-mail: chauvie@to.infn.it).

S. Guatelli, B. Mascialino and M. G. Pia are with INFN Sezione di Genova, 16146 Genova, Italy, (e-mail: Susanna.Guatelli@ge.infn.it, Barbara.Mascialino@ge.infn.it, MariaGrazia.Pia@ge.infn.it).

P. Rodrigues and A. Trindade are with the Laboratório de Instrumentação e Física Experimental de Partículas, 1000-149 Lisboa, Portugal, (email:psilva@lip.pt, andreia@lip.pt).
Electromagnetic package), and G4PenelopeBremsstrahlung, based on the physics models [9] originally developed for the Penelope [10] Monte Carlo code.

The Standard Electromagnetic package includes one process for electron Bremsstrahlung, G4eBremsstrahlung; its novel implementation [11] of cross sections has copied the approach originally devised by the Low Energy Electromagnetic package, based on the EEDL data library; the angular distribution is sampled according to a modified Tsai algorithm.

\section{METHOD OF THE STUDY}

A wide account of experimental measurements of electron Bremsstrahlung is available in literature, resulting from over 100 years of activity in this domain. Not all the available experimental data are suitable for the validation of a Monte Carlo simulation code: in some cases the reference publications do not provide sufficient details on the experimental set-up to reproduce it in the simulation with adequate accuracy for a data comparison; in other cases, especially concerning relatively old articles, the discussion of the errors affecting the measurements is not articulated enough for the purpose of the validation of simulation models.

The Bremsstrahlung experiments fall into two categories: thin-target and thick-target. Thin-target Bremsstrahlung concerns the radiation of a photon in the single collision of an electron with an atom; experiments in this category typically measure double differential cross sections. Thick-target Bremsstrahlung involves a multiple interaction process, concerning not only the radiation of a photon, but also a significant energy loss of the electron in the target; experiments of this type typically measure a yield.

A systematic, thorough validation of Geant4 Bremsstrahlung models is expected to be a complex, longterm project. This paper describes the first cycle of this study, which focuses on the low energy range.

An initial set of reference experimental data [12]-[14] has been considered for a first comparison with Geant4 simulation distributions. For this purpose a Geant4-based simulation application has been developed to reproduce the experimental conditions: the set-up consists of an electron beam of variable energy impinging on a slab of material of desired composition and thickness; different Bremsstrahlung modeling options can be selected in the simulation execution. The results of the simulation are stored in AIDA [15] analysis objects for further elaboration. 


\section{FIRST RESULTS}

The double-differential cross sections concerning the comparisons with [12] are shown in Fig. 1 to Fig. 10. The agreement is in general satisfactory, especially considering that the experimental errors discussed in [12] are significant in some cases. It is worth noticing the great similarity of the "Standard" and "Low Energy - Tsai" distributions: from the respective references, it results that the two processes adopt the same modeling approach, nevertheless they apparently duplicate code implementations, instead of exploiting the capabilities of the object oriented technology, which facilitates the code reuse through established design techniques. It is easily predictable that code duplication in a complex software system like Geant4 will be the source of maintainability problems in the future. The adoption of a more sound design in the Geant4 electromagnetic domain would be desirable to address the reuse of physics modeling functionality, at the same time preserving Geant4 fundamental feature of physics transparency.

Fig. 11 to Fig. 15 show the differential Bremsstrahlung cross section resulting from $500 \mathrm{keV}$ electrons impinging onto an aluminum target of thickness $0.548 \mathrm{~g} / \mathrm{cm}^{2}$; the corresponding experimental data are from [13]. The $2 B N$ angular distribution generator available in the Low Energy Electromagnetic package exhibits the best agreement: in fact, as documented in [7], this model is especially motivated by a precise description of the Bremsstrahlung process at low energies. The evident disagreement of the simulation results activating the Low Energy - Penelope Bremsstrahlung should be the object of further investigation.

The Low Energy - Penelope Bremsstrahlung process provides, instead, results in good agreement with the data in [14], as shown in Fig. 17.

\section{CONClusion}

The first results of a project for the systematic validation of Geant4 Bremsstrahlung models against experimental data are documented in this paper.

In most cases a satisfactory agreement is achieved between the Geant4 simulation results and the reference data; as expected on theoretical ground, the $2 B N$ angular generator model of the Low Energy Electromagnetic packages exhibits the best agreement at low energies.

Further investigation is necessary to evaluate in detail some features of the reference data, like the systematic errors they are affected by. A statistical comparison of the simulated and experimental is in progress, and will be further refined on the basis of the improved understanding of the experimental references, to provide a quantitative accounting of the simulation compatibility with experimental data.

The validation process documented in this paper has contributed to evaluate not only the accuracy of the physics modeling features of Geant4 electromagnetic processes, but it has also highlighted software design features worth to be improved.

\section{ACKNOWLEDGMENT}

The authors thank the Geant4 Collaboration for the support to their scientific activity and valuable discussions.

\section{REFERENCES}

[1] S. Agostinelli, J. Allison, K. Amako, J. Apostolakis, H. Araujo, P. Arce, et al., "Geant4 - a simulation toolkit", NIM A, vol. 506, no. 3, pp. 250303, 2004.

[2] J. Allison, K. Amako, J. Apostolakis, H. Araujo, P. Arce Dubois, M. Asai, et al., "Geant4 developments and applications", IEEE Trans. Nucl. Sci., vol. 53, no. 1, pp. 270-278, Feb. 2006.

[3] K. Amako et al., Comparison of Geant4 electromagnetic physics models against the NIST reference data, IEEE Trans. Nucl. Sci.,vol. 52, no. 4, pp. 910-918, Aug. 2005.

[4] S. Chauvie, S. Guatelli, V. Ivanchenko, F. Longo, A. Mantero, B. Mascialino, et al., "Geant4 Low Energy Electromagnetic Physics", in Conf. Rec. 2004 IEEE Nucl. Sci. Symp. Vol. 3, pp. 1881 - 1885, Oct. 2004.

[5] J. Apostolakis, S. Giani, M. Maire, P. Nieminen, M.G. Pia, L. Urban, "Geant4 low energy electromagnetic models for electrons and photons", INFN/AE-99/18, Sep. 1999.

[6] S.T. Perkins et al., "Tables and Graphs of Electron-Interaction Cross Sections from $10 \mathrm{eV}$ to $100 \mathrm{GeV}$ Derived from the LLNL Evaluated Electron Data Library (EEDL)", UCRL-50400 vol. 31, 1997.

[7] P. Rodrigues, R. Moura, C. Ortigao, L. Peralta, M.G. Pia, A. Trindade, J. Varela, "Geant4 Applications and Developments for Medical Physics Experiments," IEEE Trans. Nucl. Sci., vol. 51, no. 4, pp. 1412-1419, Aug. 2004.

[8] GEANT, CERN Program Library Long Writeup W5013, Mar. 1994.

[9] J. Sempau, J. M. Fernández-Varea, E. Acosta, and F. Salvat, "Experimental benchmarks of the Monte Carlo code PENELOPE", NIM $B$, vol. 207, no. 2 , pp. 107-123, Jun. 2003.

[10] Penelope - A code system for Monte Carlo simulation of electron and photon transport, Workshop Proceedings Issy-les-Moulineaux, France, 5-7 November 2001, AEN-NEA.

[11] "Geant4 Physics Reference Manual", [Online]. Available: http://cern.ch/geant4.

[12] N. Starfelt and H. W. Koch, "Differential Cross-Section Measurements of Thin-Target Bremsstrahlung Produced by 2.7- to 9.7-MeV Electrons", Phys. Rev., vol. 102, pp. 1598-1612, Jun. 1956.

[13] W.E. Dance et al., "Bremsstrahlung Produced in Thick Aluminum and Iron Targets by 0.5 to $2.8 \mathrm{MeV}$ Electrons", J. Appl. Phys., vol. 39, pp. $2881,1968$.

[14] R. Ambrose, D. L. Kahler, H. E. Lehtihet and C. A. QuarleS, “Angular dependence of thick-target bremsstrahlung", NIM B, vol. 56-57, pp. 327329, 1991.

[15] G. Barrand, P. Binko, M. Donszelmann, A. Johnson, A. Pfeiffer, "Abstract interfaces for data analysis: component architecture for data analysis tools", Proc. of CHEP 2001 Int. Conf. on Computing in High Energy and Nuclear Physics, pp. 215-218, Science Press, Beijing, 2001.

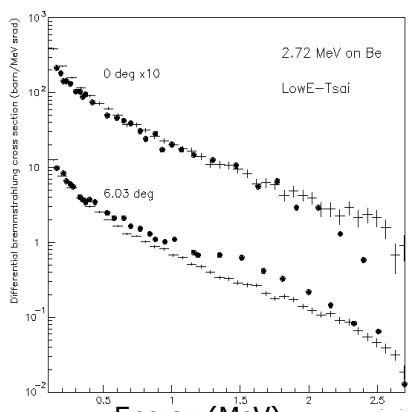

Energy (MeV)

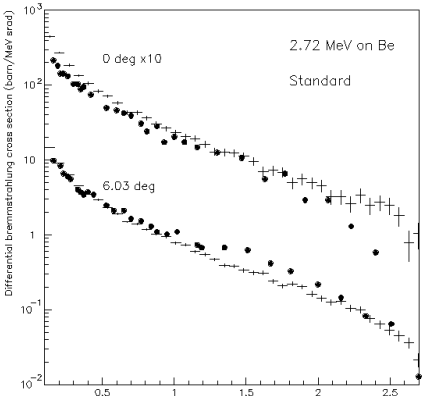

Energy (MeV)
Fig. 1. Double differential Bremsstrahlung cross section as a function of the photon energy; black circles are data in [12], crosses are Geant4 simulation results. 


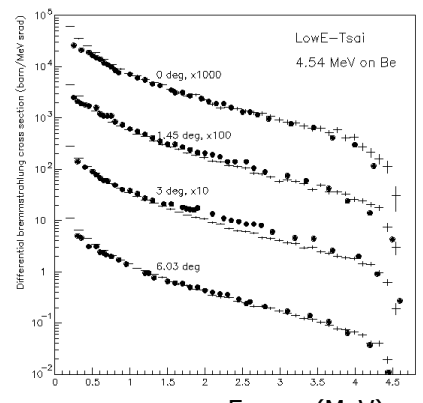

Energy (MeV)

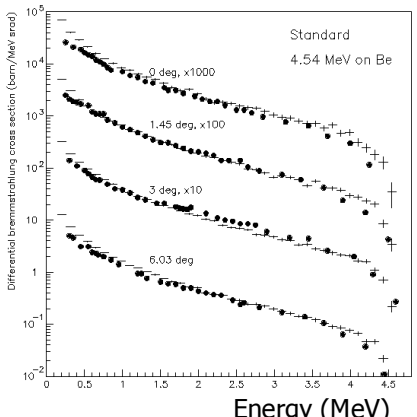

Fig. 2. Double differential Bremsstrahlung cross section as a function of the photon energy; black circles are data in [12], crosses are Geant4 simulation results.
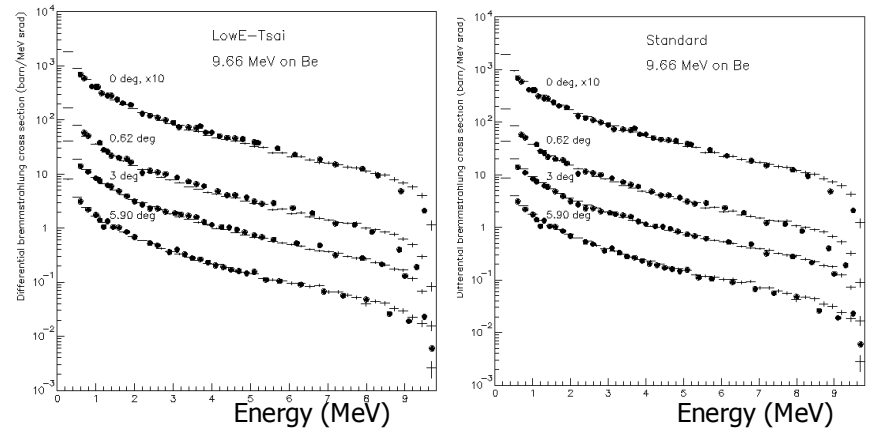

Fig. 3. Double differential Bremsstrahlung cross section as a function of the photon energy; black circles are data in [12], crosses are Geant4 simulation results.
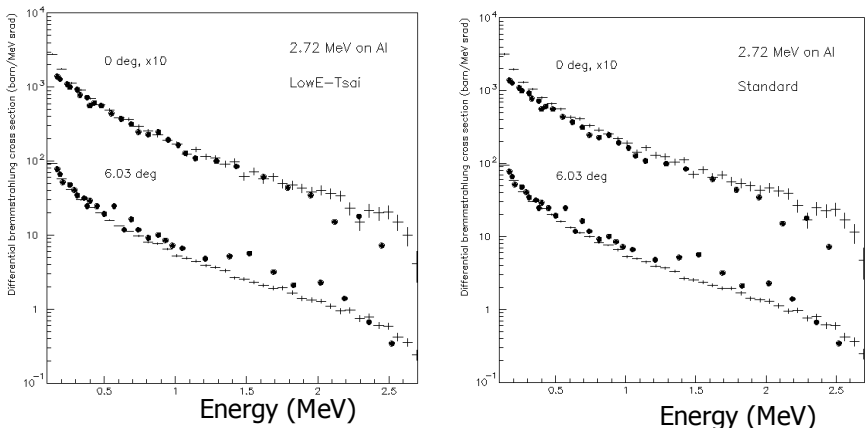

Fig. 4. Double differential Bremsstrahlung cross section as a function of the photon energy; black circles are data in [12], crosses are Geant4 simulation results.
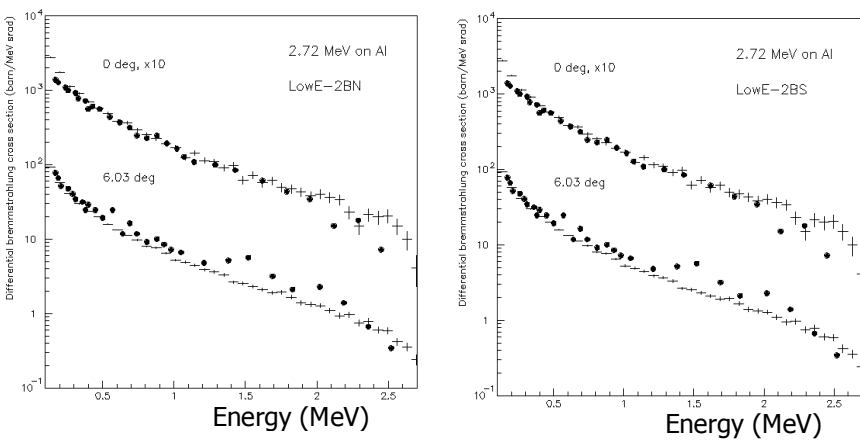

Fig. 5. Double differential Bremsstrahlung cross section as a function of the photon energy; black circles are data in [12], crosses are Geant4 simulation results.
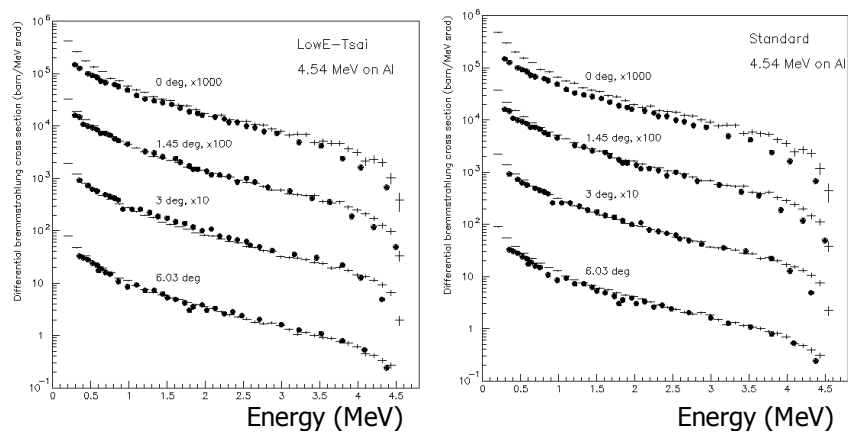

Fig. 6. Double differential Bremsstrahlung cross section as a function of the photon energy; black circles are data in [12], crosses are Geant4 simulation results.
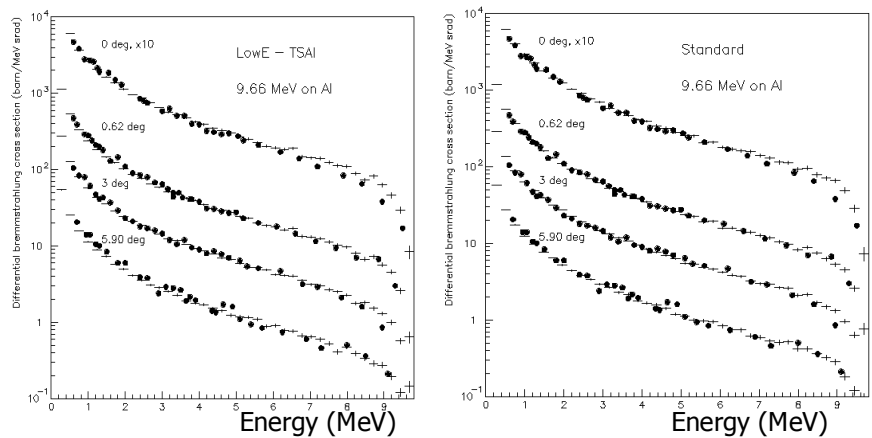

Fig. 7. Double differential Bremsstrahlung cross section as a function of the photon energy; black circles are data in [12], crosses are Geant4 simulation results.
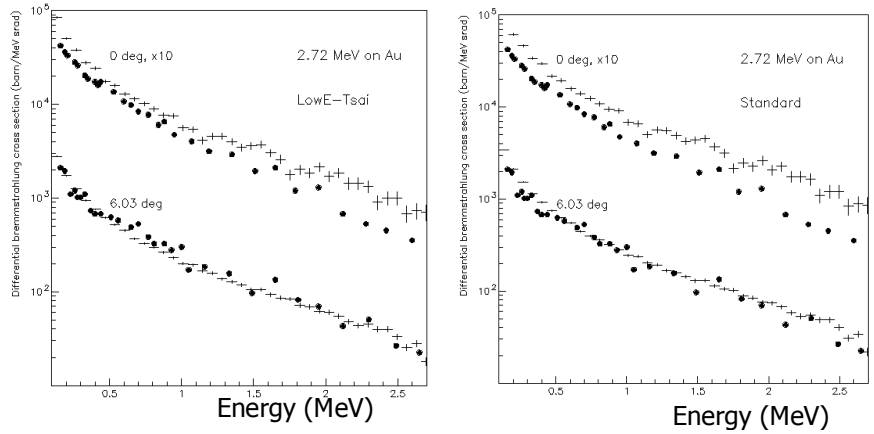

Fig. 8. Double differential Bremsstrahlung cross section as a function of the photon energy; black circles are data in [12], crosses are Geant4 simulation results.
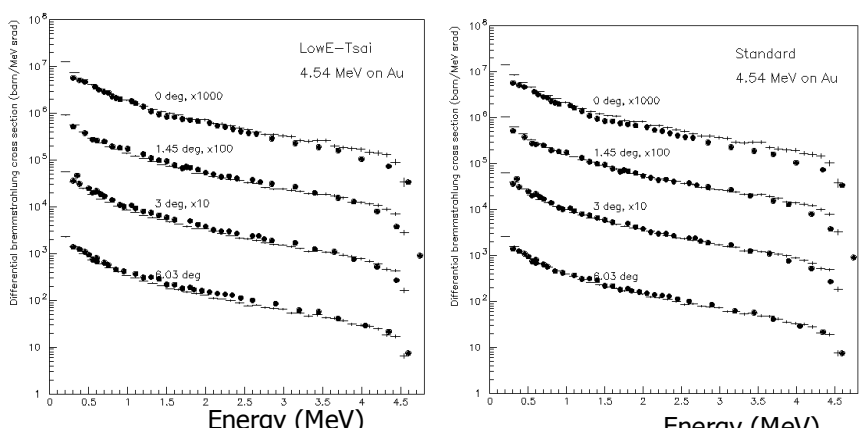

Energy (MeV)

Fig. 9. Double differential Bremsstrahlung cross section as a function of the photon energy; black circles are data in [12], crosses are Geant4 simulation results. 

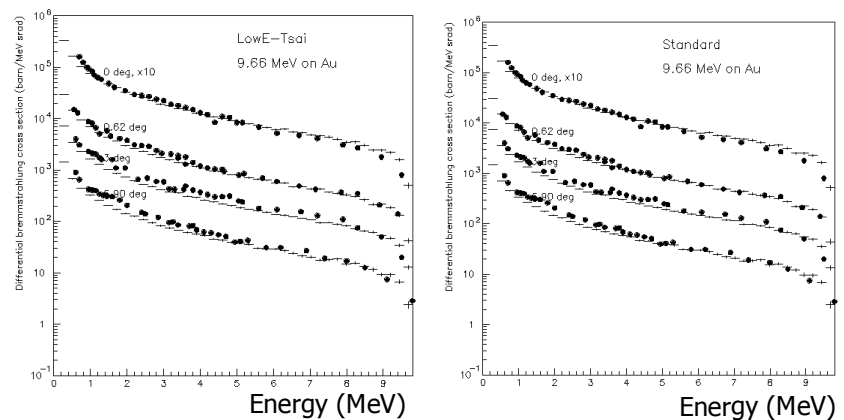

Fig. 10. Double differential Bremsstrahlung cross section as a function of the photon energy; black circles are data in [12], crosses are Geant4 simulation results.

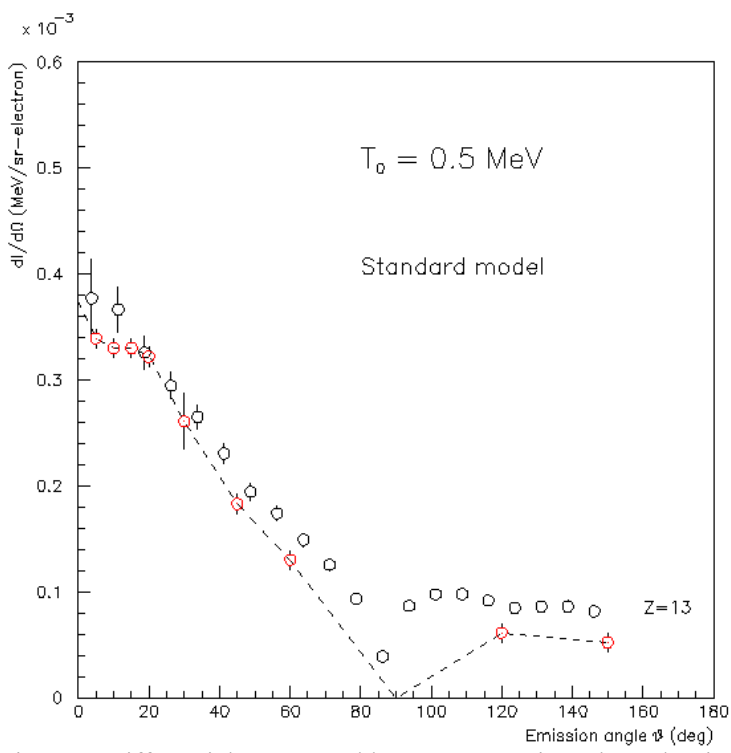

Fig. 11. Differential Bremsstrahlung cross section; the red points are data in [13] for an $\mathrm{Al}$ target, the black ones are Geant4 simulation results.

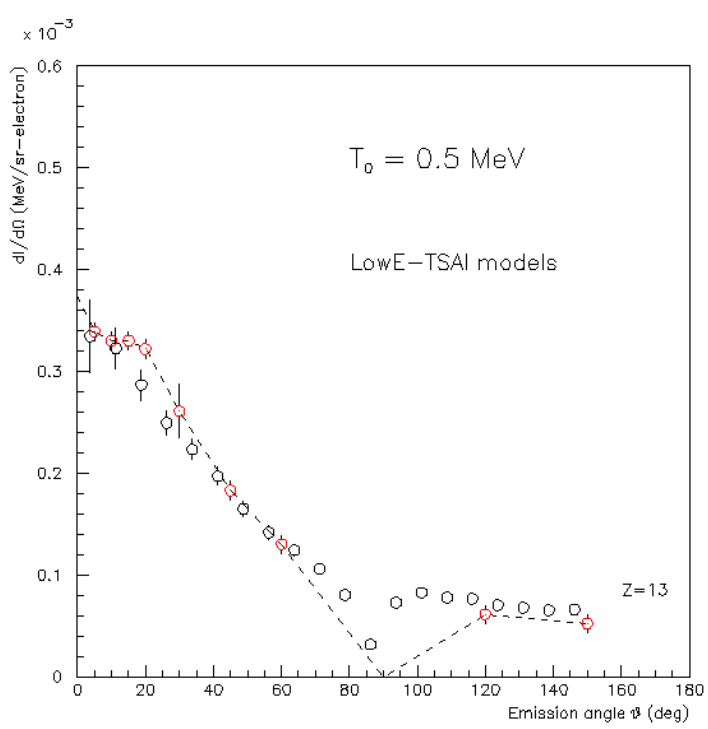

Fig. 12. Differential Bremsstrahlung cross section; the red points are data in [13] for an $\mathrm{Al}$ target, the black ones are Geant4 simulation results.

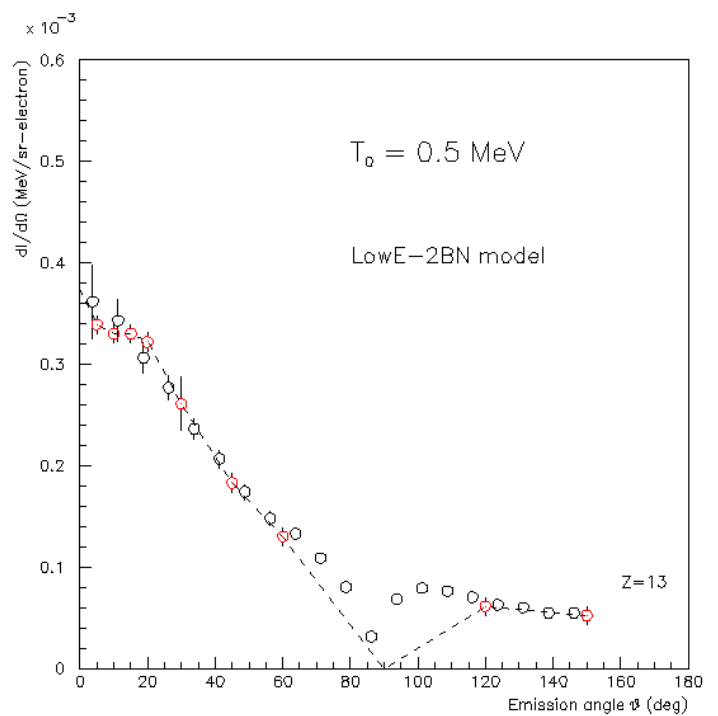

Fig. 13. Differential Bremsstrahlung cross section; the red points are data in [13] for an $\mathrm{Al}$ target, the black ones are Geant4 simulation results.

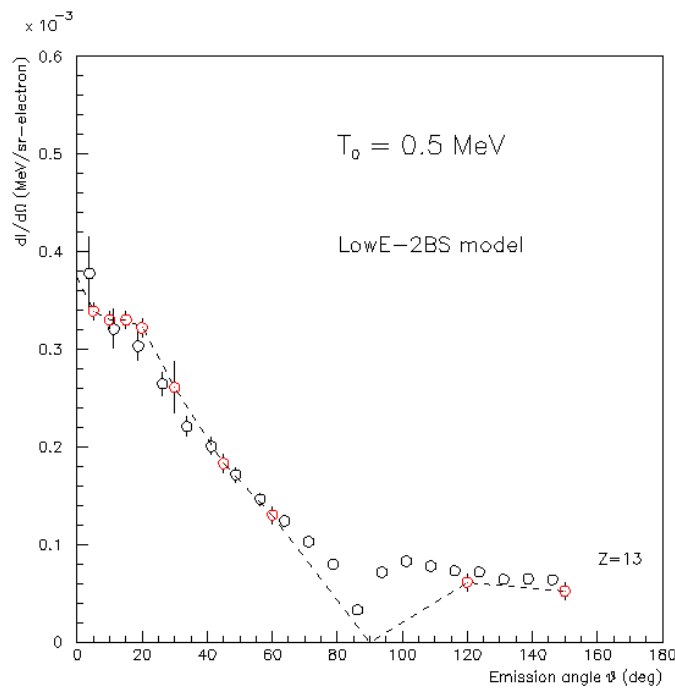

Fig. 14. Differential Bremsstrahlung cross section; the red points are data in [13] for an $\mathrm{Al}$ target, the black ones are Geant4 simulation results.

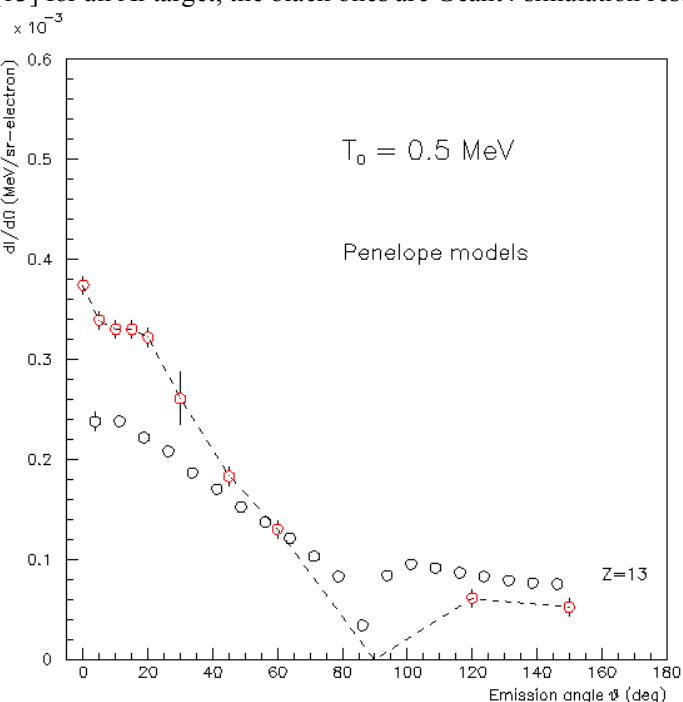

Fig. 15. Differential Bremsstrahlung cross section; the red points are data in [13] for an $\mathrm{Al}$ target, the black ones are Geant4 simulation results. 


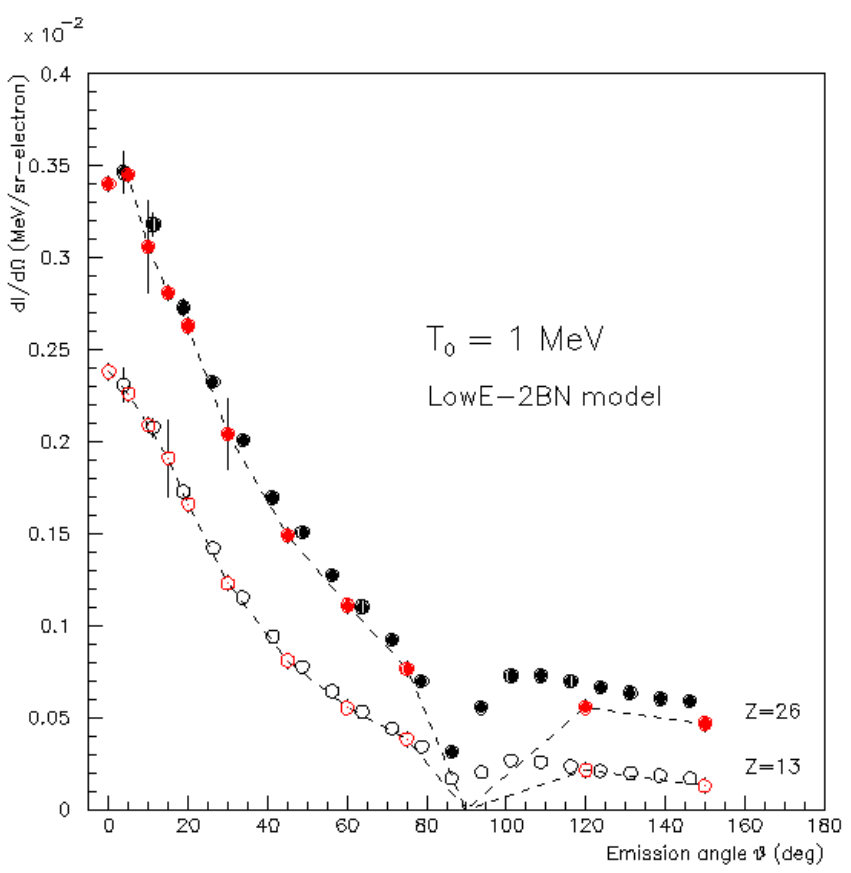

Fig. 16 Differential Bremsstrahlung cross section; the red points are data in [13], the black ones are Geant4 simulation results; the empty symbols correspond to an $\mathrm{Al}$ target, the solid ones to a Fe target.

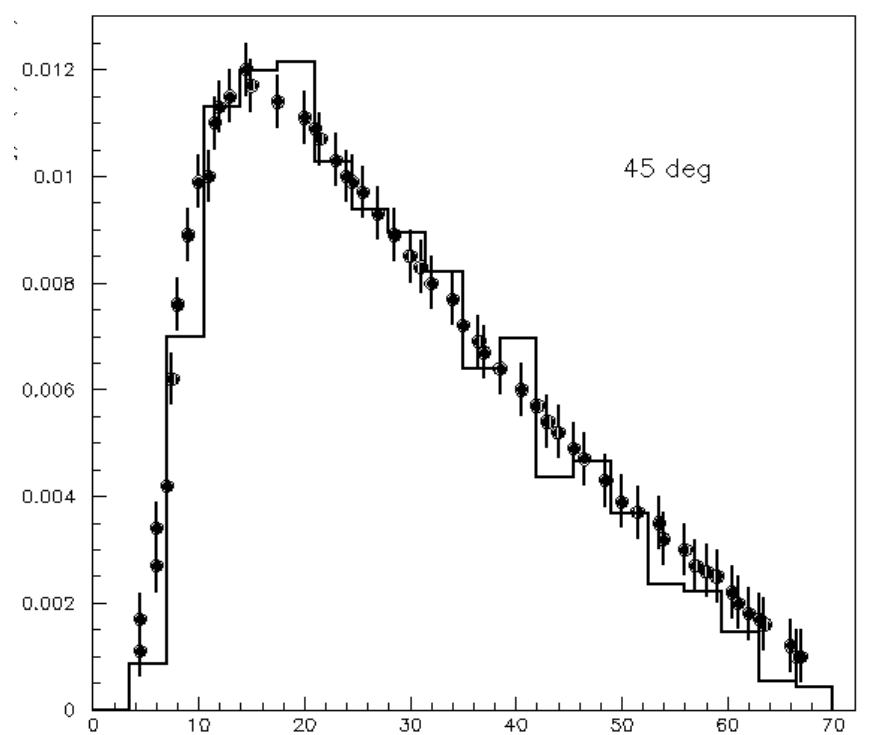

Fig. 17. Yield in eV radiated per sr per keV per incident electron scaled by $\mathrm{Z}$ versus radiated photon energy; the dots are data in [14] for $70 \mathrm{keV}$ electrons hitting an Al target; the histogram is the Geant4 simulation with the Low Energy Penelope Bremsstrahlung process. 